\title{
Continuous harmonic analysis and power quality measurements in three-phase systems
}

\author{
J.C. Montaño ${ }^{1}$, A. López ${ }^{2}$, J. Gutierrez ${ }^{3}$, M. Castilla ${ }^{4}$, D. Borrás ${ }^{4}$ and J.C. Bravo ${ }^{4}$ \\ ${ }^{1}$ Instituto de Recursos Naturales de Sevilla (Consejo Superior de Investigaciones Científicas) \\ Reina Mercedes Campus \\ P.O.B. 1052, 41080-Sevilla, (Spain) \\ phone:+34 954 624711, fax:+34 954 624002, e-mail: montano@irnase.csic.es \\ ${ }^{2}$ Department of Electronic Technology \\ E.U.P., University of Sevilla \\ Virgen de Africa, 7, 41011-Sevilla (Spain) \\ phone:+34 954 556980, fax:+34 954 282777, e-mail: alojeda@us.es \\ ${ }^{3}$ Deprtment of Applied Physics III \\ E.T.S.I.I, University of Sevilla \\ Avda. de los Descubrimientos s/n, 41092-Sevilla (Spain) \\ phone:+34 954 556980, fax:+34 954 282777, e-mail: jaiben@us.es \\ ${ }^{4}$ Department of Electrical Engineering \\ E.U.P., University of Sevilla \\ Virgen de Africa, 7, 41011-Sevilla (Spain) \\ phone:+34 954 556980, fax.+34 954 282777, e-mail: castilla@us.es,_borras@platero.eup.us.es, carlos_bravo@us.es
}

\begin{abstract}
A virtual instrument, named Power Quality Meter, is presented for (a) measuring power consumption and harmonics in three-phase systems, under non-sinusoidal and imbalance conditions (b) detecting, classifying and organizes power disturbance events. Measurement of the power consumption follows the formulation proposed by the members of the IEEE Working Group on Nonsinusoidal Situations (1996). So, definitions are based on the analysis of functions in the frequency domain, separating the fundamental terms from the harmonic terms of the Fourier series. The virtual instrument has been developed too for monitoring and measuring power disturbances, which are automatically classified and organized in a database while they are being recorded. Software tools use the database structure to present summaries of power disturbances and locate an event by severity or time of occurrence. Records of actual measurements are included to demonstrate the versatility of the instrument.
\end{abstract}

\section{Key words}

Power quality; signal processing; harmonics; data processing.

\section{Introduction}

The increased utilization of nonlinear power electronics coupled with the more prevalent use of more sensitive computer-controlled and microprocessor-based equipment has brought power quality issues on the distribution network to the forefront. Two aspects are contained in what it is named power quality: (a) the alteration of the supply signal: sinusoidal waveform, amplitude and frequency, and (b) the lack of continuity in the supply voltage (long or short interruptions). The alteration of the supply signal refers to the lack of purity of the sinusoidal waveform. This is caused, among other factors, by the harmonic content, impulsive or oscillatory transients and surges of short duration. The most interesting case, for its growing importance, it is that of, produced generally by non-linear loads (power converters, ovens of arch, transformers, rotation machines, ..).

As for the continuity in the signal supply, the quality refers to the issue of reliability about momentary interruptions and under voltages (sags). This aspect is the most important from a general point of view, including brief interruptions during less than a cycle. The number of these disturbances and their duration is the essential issue of perception by the consumer for calibrating reliability. The producer (electrical utility) is fully responsible for ensuring the best and most stable supply possible. Thus, the state of the supply signal must be monitored continuously, heeding current legislation [1].

In this environment, the power quality analysis strategy used in the instrument we have designed has been divided into those which address steady-state concerns, such as harmonic distortion or voltage imbalance, and 
transient concerns, like those resulting from faults or switching transients. Fourier spectral analysis and symmetrical component techniques are applied to the former class of problems. Threshold techniques are used for the latter, which permits on-line classification and organization of power disturbance events as they are being recorded.

Harmonics, by definition, occur in the steady state, and are integer multiples of the fundamental frequency [2]. The waveform distortion that produces the harmonics is present continually or at least for several seconds. Transients are usually dissipated within a few cycles. Transients are associated with changes in the system such as switching a capacitor bank. Harmonics are associated with the continuing operation of a load. Usually, the higher-order harmonics (above the range of the 25th to 50th, depending on the system) are negligible for power system analysis. While they may cause interference with low-power electronic devices, they are usually not damaging to the power system. It is also difficult to collect sufficiently accurate data to model power systems at these frequencies.

Harmonic distortion is blamed for many power quality disturbances that are actually transient. A measurement of the event may show a distorted waveform with obvious high-frequency components. Although transient disturbances contain high-frequency components, transients and harmonics are distinctly different phenomena and are analyzed differently. Transient waveforms exhibit the high frequencies only briefly after there has been an abrupt change in the power system. The frequencies are not necessarily harmonics; they are whatever the natural frequencies of the system are at the time of the switching operation. These frequencies have no relation to the system fundamental frequency.

Having made the distinction between the above phenomena, we have to note that meaningfully measurements of electrical quantities are obtained using the FFT algorithm applied to sampled signals in time spans corresponding to stationary events [3-5]. Further calculations perform harmonic analysis between two transient events, averaging equivalent samples over successive cycles of the voltage and current three-phase signals. This allows calculation on one representative cycle of signals captured during stationary time intervals.

Clearly, the proposed equipment provides an invaluable research tool for use in the power system research laboratories of power supply utilities, high power consumers and universities. It has been tested in real conditions, having shown its capacity to carry out an exhaustive analysis of the electric supply. All those aspects that could be included in the power quality concept [6-9] have been considered.

\section{General Specifications}

Summarized specifications of the equipment are included in Tables I and II. Table I shows a group of electrical quantities based on FFT calculation of $h$ harmonics spectra $(h=0,1, . ., 63)$. Table II shows different types of disturbances, which are detected and classified by the equipment.

\section{A.. Electrical Quantity Measurement}

Voltage and current waveforms derived from the electric line are simultaneously sampled and converted. Then, the equipment permits the instantaneous- frequency calculation of the voltage signal, which is necessary to avoid errors due to lack of synchronization between the signal period and the sampling sequence. Thus corrected samples of the set of input signals are obtained for further digital processing based on the FFT. It permits to obtain the rms value of the first 63 harmonics and the DC component. Then, harmonic components of the active and reactive powers are also obtained. All these data are necessary for obtaining the electric quantities of Table I, which have been studied extensively [3-5], [10-13]:

- RMS values of the voltage signals $\left(V_{a}, V_{b}, V_{c}\right)$ and current signals $\left(I_{a}, I_{b}, I_{c}\right)$ and corresponding equivalent values: $V_{e}$ and $I_{e}$

- Symmetrical components of the three-phase fundamental voltage $\left(V^{+}, V, V^{0}\right)$ and current $\left(I^{+}, I^{-}, I^{0}\right)$ components.

- Active and reactive powers corresponding to the fundamental components.

- Active and reactive powers corresponding to the threephase system: total active power $(P)$, positivesequence fundamental active-power $\left(P_{l}^{+}\right)$; and positive-sequence fundamental reactive-power $\left(Q_{l}{ }^{+}\right)$.

- Equivalent apparent-power, $S_{e}$, of the three-phase system.

- Unbalance Factor of phase voltages $V U F$ and current IUF.

- Total Harmonic Distortion Factor of phase voltages and line currents $T H D V$ and $T H D I$, respectively.

These quantities can be displayed before storing in the HD. The program can detect the scan backlog, that is, the processing rate versus the storing data rate, generating an index. If this index increases above a limit the program stop until new conditions permit new data processing. This operation restricts saturation and blocking of the processor.

The relative uncertainties that affect the determination of the frequency-domain components and equivalent quantities have been determined experimentally. The uncertainty in the measurement of the amplitude of the frequency-domain components was found to be less than $0.2 \%$ at the fundamental frequency and less than $3.5 \%$ at the higher harmonic frequency.

The relative uncertainties in the measurement of the equivalent quantities were found to be less than $1 \%$. Finally, the relative uncertainty in the measurement of instantaneous voltage values for disturbances detection was found to be less than $0.2 \%$. 


\section{B. Detection of disturbances}

Other specifications of the equipment, not included in Table I, refer to electrical disturbances, i.e., detection and classification of disturbances according to types shown in Table II, and further storing for monitoring and statistical analysis.

RMS events (such as sags and swells) and wave shape faults are captured by comparing the RMS calculations on successive cycles. If the RMS value changes by more than 2.5 volts RMS the wave shape change is triggered and stored. Each sampled point and the RMS values on successive cycles are stored until the wave shape stabilizes at a steady state for two cycles, designating the end of the event. A maximum number of 128 individual waveshape cycles can be stored and displayed. If RMS has not stabilized after 2 seconds, the wave shape information is discarded and then the RMS values are used to display the event. The period when the RMS value and the wave shape is changing from one stable value to the next, is defined as a transition event. The instrument classifies events either as steady state events, or transition events, depending on the rate of change of voltage. Two wave shape transition events define the start and stop of a RMS event.

To understand the relationship between the various types of events captured by the instrument, imagine an RMS fluctuation as described in Figure 1. At time to when monitoring begins, the RMS voltage is 220 volts. At time $t 1$ the voltage sags to 90 volts at time $t 2$, where it remains for steady for a period of time until $t 3$. RMS voltage then swells to 220 volts RMS again at time $t 4$. When the monitor begins monitoring there is a steady state event in progress, event 1 . Between the RMS change from the steady state of 220 volts to the steady state of 90 volts there is a transition event ( $t 1$ to $t 2$ ), event 2 , and another transition event, event 4 , from $t 3$ to $t 4$. In this example the instrument will store the 5 events as a continuous RMS plot on a single graph.

The instrument will also store all of the individual sample points during the transition events 2 and 4, and record the wave shape changes. The instrument also records long term summary displays of all RMS and power calculations.

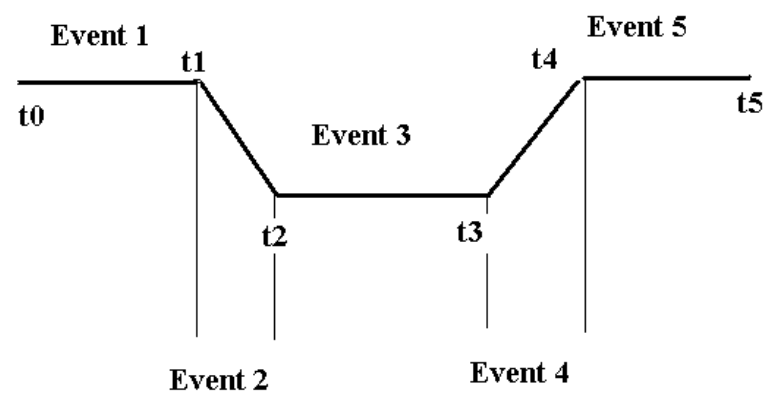

Fig. 1. RMS Events
The PQM can detect and classify the kind of disturbances shown in table II. They can be detected during a preset measurement interval, which ranges between seconds and months.

One useful characteristic of the PQM is the possibility of modifying the reference parameters used for classifying the disturbance, i.e. threshold amplitudes and intervals.

\section{Hardware}

We have constructed a three-phase analyzer to operate on line, with six channels - three of voltage and three of current. The PQM is a device that consists (Fig 2) of six transducers: three of voltage (SV) and three of current (IF), a card of data acquisition (ADA) adapted to a personal computer (PC) and a program of control, installed in the PC. SV and IF sensors are built with Hall Effect voltage and current transducers, type LV 25-P and LA25-NP respectively. The ADA device consists of a PCI-MIO 16E-4 of National Instruments, inserted in the expansion bus of the PC. It can acquire eight differential inputs using CMOS analog input multiplexers with overvoltage protection. Analog inputs are converted with 12 bits of resolution, sampled at $1.25 \mathrm{MS} / \mathrm{s}$. The PC uses a Pentium II processor at $400 \mathrm{Mhz}, 64 \mathrm{Mb}$ of RAM and a hard disk type HD $10 \mathrm{~Gb}$ Ultra DMA for data storing (ME). The device is connected to the mains to measure the three-phase quantities given in Table I. All the measures are made cycle to cycle. The instantaneous frequency is calculated with a precision of the $0,02 \%$, within the margin of 40 to 60 Hertz.

By means of a virtual switch the program changes of the 'Voltage Mode' to the 'Current Mode'. All the system components, general functions (collection of information, disk storing, etc.) and disturbance detection are controlled by the PC.

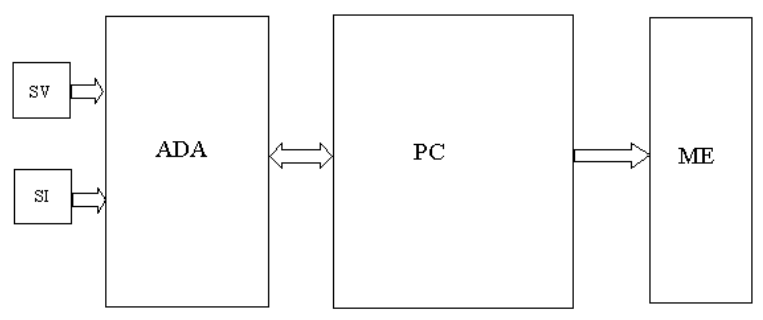

Fig. 2. PQM system.

\section{Software}

A graphical programming language (LabVIEW 5.0) is used to create the user interface that gives interactive control of the software system. LabVIEW is integrated with the ADA board for data development, analysis and presentation solutions.

The measurement results are stored in different files according to the type of information recorded: 
- Instantaneous voltage and current values of input signals. They are shown in the captured screen of figure 3.

- $\quad$ Positive, negative and zero sequences derived from the input signals (Fig 3).

- Spectra of voltage and current signals in the three wires (Fig. 3).

- Electrical quantities (Table I) of voltage and current signals defined in separated conductors of the three-phase system, and electrical quantities defined for the three-phase system (equivalent values).

- $\quad$ Type and number of disturbances.

- Instantaneous voltage and current values of cycles containing disturbances, two previous and two hind cycles (Fig 4).

Instantaneous disturbances occurring within a fundamental period (oscillatory transients, notching, impulses, etc.) are processed differently. To classify this type of disturbance, each of the voltage samples of the cycle in process are compared with those of a standard cycle. This can detect the correct allocation of the sample inside the preset band. The width of this band is set initially when the measuring period is configured. The standard cycle of voltage samples is also obtained in the initial cycles of operation of the system and it is actualized in each of the following cycles.

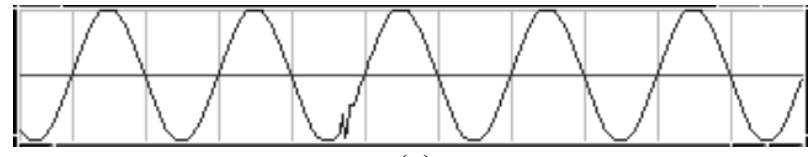

(a)

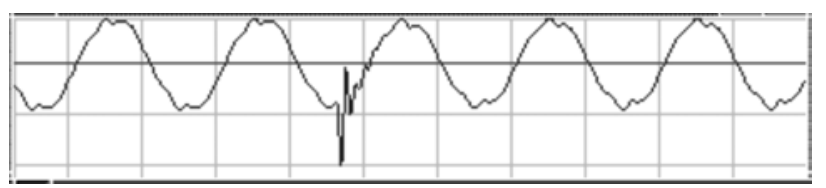

(b)

Fig, 4. Instantaneous (a) voltage and (b) current values containing a transition event.

\section{Conclusion}

The PQM system has been tested in real conditions, having shown its capacity to carry out an exhaustive analysis of the electric supply. All those aspects that could be included in the power quality concept have been considered, in particular: (a) measuring power consumption and harmonics in three-phase systems, under non-sinusoidal and imbalance conditions (b) detecting, classifying and organizes power disturbance events.

\section{Acknowledgement}

This work was derived from a research project among the University of Seville and the Council for Scientific Research, and is included in the Program of Investigation of the Spanish Ministry of Science and Technology.

\section{References}

[1] International Electrotechnical Commission. "Disturbances in supply system caused by household appliances and similar electrical equipment. Part 1: Definitions, Part 2: Harmonics, Part 3: Voltage fluctuations." Publication 555 (555-1, 555-2, 555-3), Geneva, 1982.

[2] J. Arrillaga, D.A. Bradley y P.S. Bodger, "Power System Harmonics", New York, John Wiley, 1985.

[3] J.C. Montaño, M.Castilla, A. López y J. Gutiérrez, "Measurement of Apparent Power Components in the Frequency Domain", IEEE Trans. Instrum. Meas., Vol. 39, No.4, 1990, pp. 583-587.

[4] A. Menchetti, R. Sasdelli, "Measurement problems in Power Quality Improvement," ETEP Vol. 4, No. 6, 1994, pp. 463-468.

[5] J.C. Montaño, A. López, M. Castilla y J. Gutiérrez, "DSPbased algorithm for electric power measurement", IEE Proceedings-A, vol. 140, pp. 485-490, 1993.

[6] T.S. Key, "Diagnosing Power Quality Related Computer Problems", IEEE Trans. on Industry Applications, Vol. IA15, No. 4, July/August 1979.

[7] E.M. Gulachenski, D.P. Symanski, "Distribution Power Quality Considerations for Supply to Large Digital Computer Loads" IEEE Trans. PAS, Vol. 100, No. 12, Dec. 1981.

[8] A. Domijan et al., "Directions of Research on Electric Power Quality", IEEE Trans. on Power Del., Vol. 8, (1993) No. 1, pp. 429-436.

[9] A. Tugulea. "Criteria for the Definition of the Electric Power Quality and its Measurement System", ETEP, Vol. 6, (1996) No. 5, pp. 357-363.

[10] IEEE Working Group on Nonsinusoidal Situations: Effects on Meter Performance and Definitions of Power, 'Practical Definitions for Powers in Systems with Nonsinusoidal Waveforms and Unbalanced Loads: A Discussion,' IEEE Trans. Power Delivery, Vol. 11, No. 1, Jan. 1996.

[11] A.E. Emanuel, 'On the definition of power factor and apparent power in unbalanced polyphase circuits with sinusoidal voltage and currents', IEEE Trans. on Power Deliv., Vol.8, No.3, July 1993.

[12] L. S. Czarnecki, 'Orthogonal Decomposition of the Currents in a 3-Phase Nonlinear Asymmetrical Circuit with a Nonsinusoidal Voltage Source,' IEEE Trans. Instrum. Meas., vol. 37, No. 1, March 1988.

[13] J.C. Montaño and P. Salmerón, "Identification of instantaneous current components in three-phase systems", IEE Proc.-Sci. Meas. Technol., Vol. 146, No. 5, pp. 227$231,1999$. 
TABLE I

ELECTRICAL QUANTITIES

Quantities corresponding to $N$ harmonics spectrum, and four conductors, $l=a, b, c, n$ (neutral).
RMS value of the phase voltages and line currents

Fundamental active and reactive powers of the phase voltages and line currents Active and reactive
powers of the phase voltages and line currents

Distortion of the phase voltages and line currents

\begin{tabular}{|c}
\hline \\
\\
Equivalent quantities \\
derived from phase
\end{tabular}
voltages and line currents

ing

Equivalent voltage

and current

\begin{tabular}{|l} 
\\
\hline \\
Active Power
\end{tabular}

$$
\begin{aligned}
& V_{h}=\left|V_{h}\right| e^{j \alpha_{h}}, \quad I_{h}=\left|I_{h}\right| e^{j \beta_{h}} \quad h=0,1, . ., N-1 \\
& V_{l}^{2}=\frac{1}{N} \sum_{h=0}^{N-1}\left|V_{l h}\right|^{2}, \quad I_{l}^{2}=\frac{1}{N} \sum_{h=0}^{N-1}\left|I_{l h}\right|^{2} \quad l=a, b, c, n
\end{aligned}
$$

e

$$
\begin{aligned}
& P_{l 1}=\left|V_{l 1}\right|\left|I_{l 1}\right| \cos \varphi_{l 1}, \quad \varphi_{l 1}=\alpha_{l 1}-\beta_{l 1} \\
& Q_{l 1}=\left|V_{l 1}\right|\left|I_{l 1}\right| \sin \varphi_{l 1} \\
& S_{l 1}^{2}=P_{l 1}^{2}+Q_{l 1}^{2}
\end{aligned}
$$$$
P=\sum_{l} P_{l}, \quad P_{l}=\sum_{h} P_{l h}=\sum_{h} V_{l h} I_{l h} \cos \varphi_{l h}
$$$$
Q=Q_{1}=\sum_{l} Q_{l 1}=\sum_{l} V_{l 1} I_{l 1} \sin \varphi_{l 1}
$$$$
T H D V_{l}=\frac{\sqrt{\sum_{h \neq 1} V_{l h}^{2}}}{V_{l 1}}, \quad \text { THDI }_{l}=\frac{\sqrt{\sum_{h \neq 1} I_{l h}^{2}}}{I_{l 1}}
$$

$$
\begin{array}{cc}
V_{e 1}^{2}=\frac{V_{a 1}^{2}+V_{b 1}^{2}+V_{c 1}^{2}}{3}, & I_{e 1}^{2}=\frac{I_{a 1}^{2}+I_{b 1}^{2}+I_{c 1}^{2}}{3} \\
V_{e}^{2}=V_{e 1}^{2}+V_{e H}^{2}, & I_{e}^{2}=I_{e 1}^{2}+I_{e H}^{2}
\end{array}
$$$$
V_{e 1}^{2}=\frac{1}{3} \sum_{l} V_{l 1}^{2}, \quad I_{e 1}^{2}=\frac{1}{3} \sum_{l} I_{l 1}^{2}
$$$$
V_{e H}^{2}=\frac{1}{3} \sum_{l h \neq 1} V_{l h}^{2}, \quad I_{e H}^{2}=\frac{1}{3} \sum_{l h \neq 1} I_{l h}^{2}
$$

(

\begin{tabular}{|c|c} 
& \\
\hline Reactive Power & \\
\hline $\begin{array}{c}\text { Equivalent Apparent } \\
\text { Power }\end{array}$ & \\
\hline & \\
\hline
\end{tabular}

Unbalance factor

Equivalent Total

Harmonic Distortion

of the phase voltages

and line currents

$V U F=\frac{V_{1}^{-}}{V_{1}^{+}}$
$T H D V_{e}=\frac{V_{e H}}{V_{e 1}}$


TABLE II

DISTURBANCES

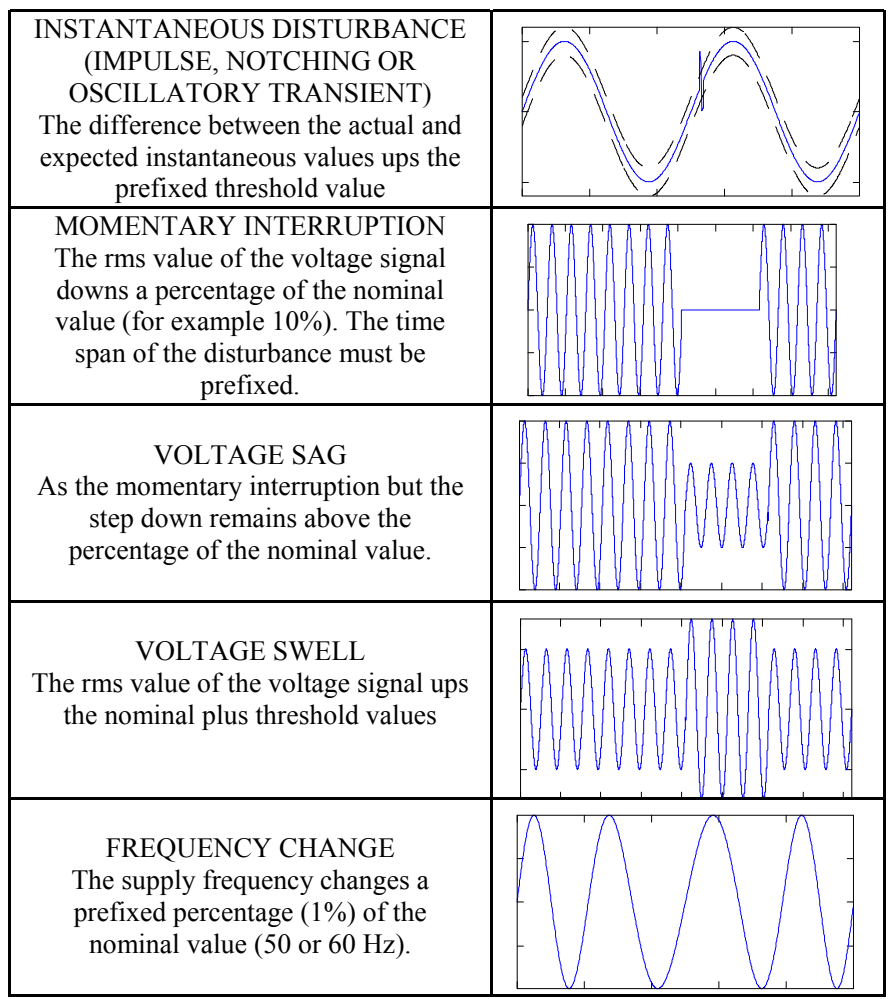

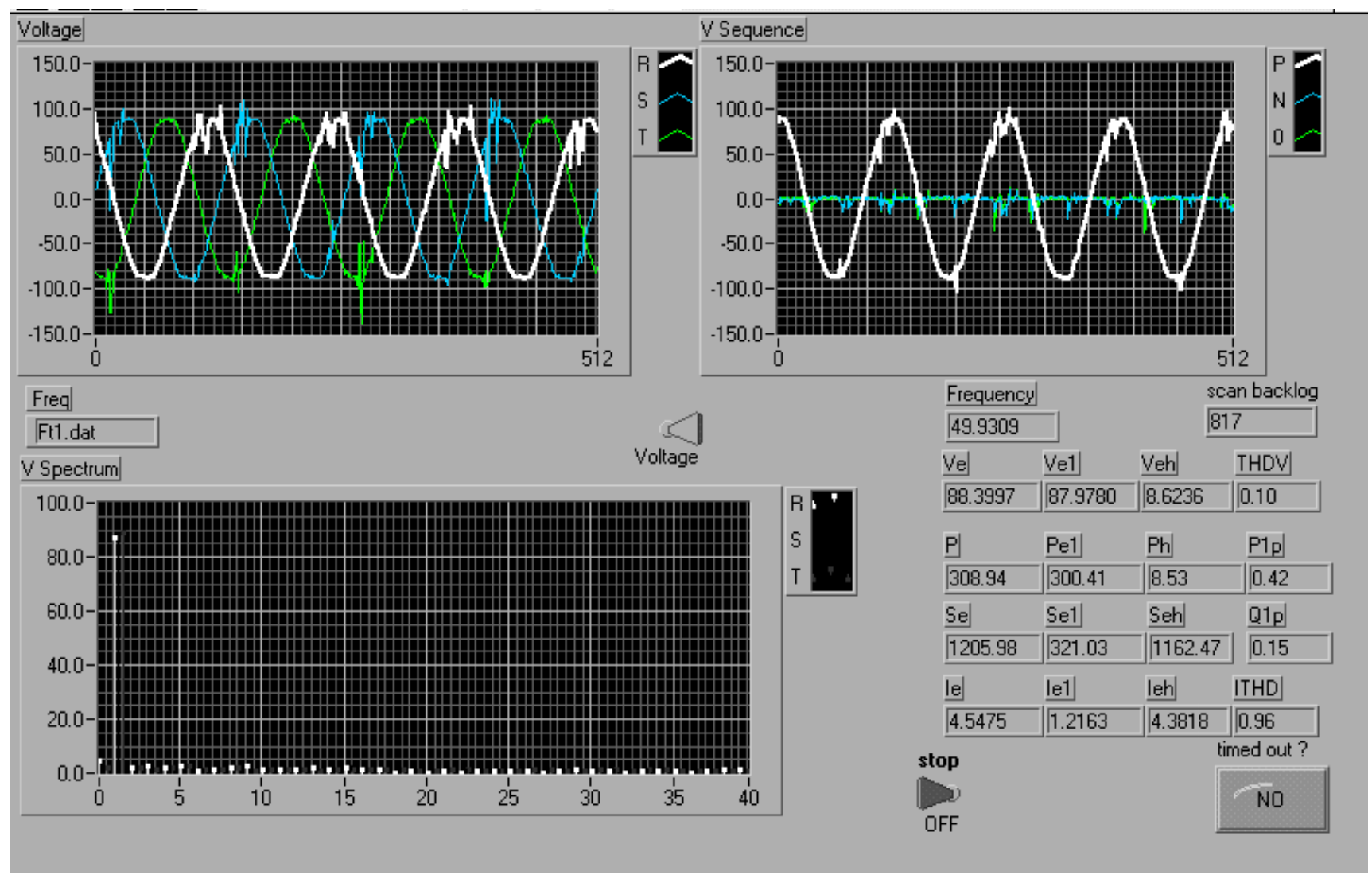

Fig.3. Captured screen of the virtual equipment. 